\title{
Electrochemically deposited silver detection substrate for surface- enhanced Raman spectroscopy cancer diagnostics
}

Zuzana Orságová Králová

Andrej Oriňak

Renáta Oriňaková

Ondrej Petruš

Ján Macko

Jozef Radoňak

Lucia Sukovská Lakyová

Zuzana Jurašeková

Roger M. Smith

Magdaléna Strečková

Karol Koval' 


\title{
Electrochemically deposited silver detection substrate for surface-enhanced Raman spectroscopy cancer diagnostics
}

\author{
Zuzana Orságová Králová, ${ }^{\mathrm{a}, \star}$ Andrej Oriňak, ${ }^{\mathrm{a}}$ Renáta Oriňaková, ${ }^{\mathrm{a}}$ Ondrej Petruš, ${ }^{\mathrm{a}}$ Ján Macko, ${ }^{\mathrm{a}}$ Jozef Radoňak,

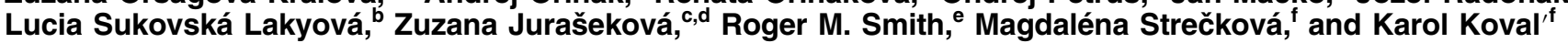 \\ aUniversity of P. J. Safarik in Kosice, Department of Physical Chemistry, Kosice, Slovakia \\ bLouis Pasteur University Hospital in Kosice, Surgical Clinic, Kosice, Slovakia \\ 'University of P. J. Safarik in Kosice, Department of Biophysics, Kosice, Slovakia \\ dUniversity of P. J. Safarik in Kosice, Center for Interdisciplinary Biosciences, Kosice, Slovakia \\ 'Loughborough University, Department of Chemistry, Loughborough, United Kingdom \\ 'Slovak Academy of Sciences, Institute of Materials Research, Kosice, Slovakia
}

\begin{abstract}
Surface-enhanced Raman spectroscopy (SERS) of blood plasma on an electrochemically prepared silver surface has been studied as a label-free, noninvasive diagnostic test for colorectal cancer. Indium tin oxide glass substrates were modified with $0.01 \mathrm{~mol} \mathrm{dm}^{-3}$ silver nitrate using the pulsed double-potentiostatic method. The prepared silver substrates were tested with Rhodamine $6 \mathrm{G}$ as a model analyte and the surface with the highest signal enhancement was selected. This silver dendritic surface was used as a diagnostic substrate for SERS measurements of human blood plasma. A group of oncological patients with declared colorectal carcinoma $(n=15)$ and the control group of healthy volunteers $(n=15)$ were compared. The biomolecular changes in chemical composition in the cancer samples were detected by statistical processing of the resulting SERS spectra. About $94 \%$ specificity and $100 \%$ sensitivity were achieved for the analysis by the ratio of the SERS peak intensity at $725 \mathrm{~cm}^{-1}$ for adenine to the peak intensity at $638 \mathrm{~cm}^{-1}$ for tyrosine and $100 \%$ specificity and sensitivity by using principal component analysis. This method of SERS diagnostics of colorectal cancer, which does not require the nanoparticle preparation, mixing, and incubation of plasma with a colloidal solution as in conventional tests, is a rapid, inexpensive method, which could be introduced as a primary diagnostic test. ๑ 2018 Society of Photo-Optical Instrumentation Engineers (SPIE) [DOI: 10.1117/1.JBO.23.7.075002]
\end{abstract}

Keywords: electrodeposition; substrates; surface-enhanced Raman spectroscopy; plasma; diagnostics.

Paper 180184RR received Mar. 27, 2018; accepted for publication Jun. 18, 2018; published online Jul. 6, 2018.

\section{Introduction}

Colorectal cancer is the third most frequently diagnosed type of cancer and the fourth leading cause of oncological diseaserelated deaths in the world. It is expected to increase by $60 \%$ to more than 2.2 million cases and 1.1 million cancer deaths by $2030 .{ }^{1}$ Current methods of colorectal cancer diagnostic include invasive colonoscopy ${ }^{2}$ and sigmoidoscopy, ${ }^{3}$ colon computer tomography ${ }^{2}$ or noninvasive biochemical ${ }^{4} /$ immunochemi- $^{-}$ cal tests of fecal material. ${ }^{5}$ Noninvasive initial tests are followed by a biopsy test and an appropriate treatment. The ideal biofluid for this type of cancer diagnostics is blood plasma, which carries information about the ongoing processes in the human organism. Individual components of plasma are sensitive to the mutagenic processes. ${ }^{6}$ These changes in the biomolecular composition of blood plasma appear as an increase in specific nucleic acids and a decrease in the saccharide and protein content of the plasma of colorectal cancer patients compared to those of healthy subjects. ${ }^{7}$ Several researchers have tried to detect these changes in cancerous blood plasma composition by Raman spectroscopy (RS) and surface-enhanced Raman spectroscopy (SERS). RS is a well-established analytical

*Address all correspondence to: Zuzana Orságová Králová, E-mail: zuzana .orsagova.kralova@student.upjs.sk technique and provides fingerprint information of the structure and conformation of macromolecules, such as proteins, nucleic acids, and lipids. ${ }^{7,8}$ It has been used as an experimental diagnostic method for the differentiation of normal and cancerous tissues from larynx,${ }^{9}$ nasopharynx,${ }^{10}$ breast, ${ }^{11}$ lung, ${ }^{12}$ cervix,${ }^{13}$ prostate, ${ }^{14}$ salivary, ${ }^{15}$ skin,,${ }^{16}$ or brain,,${ }^{17}$ as well as other diseases, such as bacterial infections, ${ }^{18}$ atherosclerosis, ${ }^{19}$ diabetes,${ }^{20}$ or even Alzheimer's ${ }^{21}$ and inflammatory diseases. ${ }^{22}$ However, RS also has its limitations as the signal intensity is often very weak and, as a result, the fluorescence of impurities or the specimen itself can hide the Raman spectrum, or the intense laser radiation can destroy the sample. ${ }^{23}$ This problem can be solved by SERS, which is a vibrational-spectroscopy technique based on the enhancement of Raman scattering by metallic nanostructures with suitable plasmonic hotspot characteristics (metal nanoparticles, ${ }^{24}$ nanorods, ${ }^{25}$ dendrites,${ }^{26}$ etc. $) .{ }^{27}$ The hotspots are localized areas of intense local field enhancement caused by local surface plasmon resonance (SPR), where the electromagnetic field is the most intense, and provide enormous signal enhancement. ${ }^{28}$ Because of this, SERS is highly suitable for detailed analysis, such as early and precise diagnostic methods for oncologic diseases. Feng et al. ${ }^{29}$ developed a method for 
the early detection of nasopharyngeal cancer from blood serum samples using SERS, which was tested in a clinical trial with 43 patients with diagnosed cancer and 33 control healthy volunteers. The untreated serum was mixed with silver colloidal particles, which were used as metal SERS active surfaces for signal intensity enhancement. The signal intensities of individual specific Raman peaks were compared using principal component analysis (PCA) and discriminated between the groups with $90.7 \%$ sensitivity and $100 \%$ specificity. ${ }^{29}$ Further studies of cervical, ${ }^{30}$ colon, ${ }^{31}$ stomach, ${ }^{32}$ parotid gland, ${ }^{33}$ and prostate $^{34}$ cancer found that the individual types of cancer had characteristic Raman peaks. The unwanted and uncontrollable aggregation of the nanoparticles can be avoided by replacing the colloids of noble metals by electrodeposited SERS substrates. The intermediate step, mixing the colloidal particles with the sample and its subsequent incubation, can then be omitted, which accelerates the whole diagnostic process.

One of the "bottom up" economical methods of preparing SERS substrates is the electrodeposition of metallic nanostructures onto an indium tin oxide (ITO) glass carrier substrate from an electrolyte. ${ }^{35,36}$ Several techniques have been employed, such as utilizing a template, ${ }^{37}$ applying double potential pulses, ${ }^{38,39}$ galvanostatic deposition, ${ }^{40}$ or a cyclic voltammetry scan. ${ }^{41}$ Less frequently pulsed double-potentiostatic deposition has been used, although it is a reliable and simple method for controlling the size and morphology of electrodeposited nanostructures by changing the potentials for the nucleation and growth phases. ${ }^{42}$ The substrate preparation can be optimized by altering the coating bath compositions, applied potentials, and the duration of individual steps. ${ }^{36}$

This paper describes the preparation of silver dendritic nanostructured films with a suitable morphology for diagnostic high sensitivity SERS substrates by optimization of the pulsed double-potentiostatic method. The resulting substrates were characterized by their high hydrophobicity without any pretreatment by alkylsilanes ${ }^{43}$ or alkanethiols ${ }^{44}$ as it is essential to fix the water-soluble samples to a specified location on the SERS substrate, because preconcentration of the sample at a fixed place is desirable for successful SERS analysis. ${ }^{45}$

The electrochemically prepared SERS substrates were tested with R6G as a model analyte, to select the substrate providing the highest signal enhancement. This material was then used in primary experiments as a superhydrophobic SERS substrate for the rapid, noninvasive, and nonlabeled diagnosis of colorectal cancer.

\section{Experimental Part}

\subsection{Chemicals and Materials}

Acetone, ethanol, and silver nitrate were obtained from Sigma Aldrich (Missouri) and used without further purification. Rhodamine 6G (R6G) was purchased from Acros Organics (New Jersey). Ultrapure $18.2 \mathrm{M} \Omega / \mathrm{cm}$ water was used for the preparation of solution and cleaning samples. ITO glass slides with $<7 \Omega / \mathrm{sq} \mathrm{cm}$ resistance were purchased from Zhuhai Kaivo Optoelectronic Technology Co., Ltd. (China). Plastic cassette to hold the SERS substrate was obtained from Jieyi Biotech Co., (Shanghai, China). Blood collection tubes with $\mathrm{K}_{3}$ EDTA as an anticoagulant were used for blood samples (BD Vacutainer, New Jersey).

\subsection{Substrate Sample Preparation}

Silver layers were electrochemically deposited onto ITO glass used as the working electrode in a three electrode electrochemical cell, with a platinum counter electrode and saturated calomel electrode (Autolab PGSTAT302N Metrohm, The Netherlands). Before the electrodeposition, ITO glass plates were cut into small pieces $(1 \times 1 \mathrm{~cm})$. They were ultrasonically cleaned for $15 \mathrm{~min}$ in each of the following solvents: acetone, ethanol, and ultrapure water. The electrolyte solution contained $0.01 \mathrm{~mol} \mathrm{dm}^{-3} \mathrm{AgNO}_{3}$ at a $\mathrm{pH}$ of 4.8 . For optimization of the most appropriate morphology for the SERS diagnostic substrate, 16 samples by pulsed double-potentiostatic method $\left(E_{1}=-0.4 \mathrm{~V}\right.$ for $\left.1 \mathrm{~s}, E_{2}=-0.1 \mathrm{~V}\right)$ at laboratory temperature were prepared. The number of $E_{2}$ pulses was varied from 10 to 70 and duration time from 0.5 to $2 \mathrm{~s}$. The area of the electrodeposited dendritic SERS surface was $0.5 \mathrm{~cm}^{2}$. After electrodeposition, the substrate sample was rinsed with ultrapure water and dried in the air.

\subsection{Testing Substrates}

Five microliters of an aqueous solution of $1 \times 10^{-6} \mathrm{~mol} \mathrm{dm}^{-3}$ R6G was pipetted onto all 16 electrochemically prepared silver surfaces as a model analyte. For concentration-dependent analysis, solutions were used with concentrations from $1 \times 10^{-6} \mathrm{~mol} \mathrm{dm}^{-3}$ to $1 \times 10^{-12} \mathrm{~mol} \mathrm{dm}^{-3}$. The diameter of the air-dried droplet of R6G was $1.7 \mathrm{~mm}$.

\subsection{Plasma Samples}

Blood plasma samples were used to examine the selected $\mathrm{Ag}$ SERS substrate for use in the detection of colorectal cancer. Samples from healthy volunteers came from a clinical diagnostics laboratory. Blood samples from female and male colorectal cancer patients, aged 35 to 75 years, came from the Surgery Department, Louis Pasteur University Hospital, in Kosice. Five milliliters of blood was collected by venipuncture into vacutainer blood collection tubes containing $\mathrm{K}_{3}$ EDTA as an anticoagulant. Blood was taken systematically between 7:00 a.m. and 7:30 a.m. and was stored at $8^{\circ} \mathrm{C}$. The plasma was separated from the blood by centrifugation at $3000 \mathrm{rpm}$ for $10 \mathrm{~min}$. The supernatant plasma was pipetted into $2 \mathrm{ml}$ Eppendorf tubes and stored at $-18^{\circ} \mathrm{C}$. For SERS measurements, $5 \mu \mathrm{l}$ of a mixture of blood plasma and ultrapure water, in a ratio $1: 9$, was dropped onto the selected silver surface and dried under ambient air condition. The diameter of air-dried droplet of water diluted blood plasma was $2.1 \mathrm{~mm}$.

\subsection{Surface Characterization Techniques}

The morphology of the silver surfaces was characterized by a scanning electron microscope (SEM; JEOL JSM 7000F, Japan). Raman measurements were carried out using a Renishaw InVia Raman microscope (Gloucestershire, United Kingdom) with an excitation wavelength at $532 \mathrm{~nm}$ for R6G analysis and $785 \mathrm{~nm}$ for analysis of human blood plasma. The laser beam was directed onto the sample through a $20 \times$ objective lens. The spectra were collected through the same objective over the range of 400 to $1800 \mathrm{~cm}^{-1}$. For R6G measurements, the integration time was set at $10 \mathrm{~s}$, three accumulations, and $0.05 \%$ of original laser power, whereas for blood plasma analysis, the integration time was set at $10 \mathrm{~s}$, three accumulations, and $1 \%$ of original laser power. SERS spectra were corrected to the baseline and 
Table 1 Values of contact angle for water, thiodiglycol, and glycerol measured on silver substrates prepared at different number of pluses and pulse duration.

\begin{tabular}{|c|c|c|c|c|c|c|c|c|c|c|c|c|}
\hline & \multicolumn{4}{|c|}{ Water } & \multicolumn{4}{|c|}{ Thiodiglycol } & \multicolumn{4}{|c|}{ Glycerol } \\
\hline & $10 p$ & $30 p$ & $50 p$ & $70 p$ & $10 p$ & $30 p$ & $50 p$ & $70 p$ & $10 p$ & $30 p$ & $50 p$ & $70 p$ \\
\hline $0.5 \mathrm{~s}$ & 115 & 136 & 153 & 155 & 42 & 31 & 16 & 64 & 90 & 85 & 77 & 77 \\
\hline $1 \mathrm{~s}$ & 117 & 132 & 147 & 153 & 49 & 49 & 30 & 81 & 96 & 93 & 86 & 81 \\
\hline $1.5 \mathrm{~s}$ & 111 & 124 & 143 & 148 & 45 & 36 & 25 & 59 & 98 & 86 & 97 & 92 \\
\hline $2 \mathrm{~s}$ & 129 & 138 & 141 & 157 & 56 & 42 & 30 & 60 & 125 & 122 & 115 & 105 \\
\hline
\end{tabular}

averaged from three scans. The concentration dependence was determined by measurement from 10 random spots for each concentration of R6G.

\subsection{Test of Contact Angles}

Contact angles were measured by dropping $6 \mu \mathrm{l}$ of water, thiodiglycol, and glycerol (Table 1) onto the silver surfaces. The droplets were captured by EO-311C USB color camera (Edmund Optics) and ImageJ software with plug-in LB-ADSA was used to determine the contact angles.

\subsection{Electromagnetic Simulation of Ag Surface}

To analyze the local electric field distribution of the dendritic structures, we performed two-dimensional finite-difference time-domain (FDTD) simulation (Lumerical FDTD Solution 8.15.786, Lumerical Inc., Canada). Simulation models consisted of single silver (Palik) dendritic structure, where the diameter and length of trunk were set to 300 and $4000 \mathrm{~nm}$, respectively. The branches consist of spheroid nanoparticles with diameter $300,250,200$, and $150 \mathrm{~nm}$ from trunk to outside, respectively. The light source was planewave (400 to $750 \mathrm{~nm})$ propagating along the $z$ direction with the polarization parallel to the $x$-axis. The boundary condition was set with periodic boundary conditions in $x$-direction and a perfectly-matched-layer boundary condition in $z$-direction. Mesh size was set to $5 \mathrm{~nm}$. The frequency-domain field profile was set to $532 \mathrm{~nm}$ in $x z$ direction.

\section{Results}

\subsection{Silver Surface Characterization by Scanning Electron Microscope}

An electrochemical deposition method was used for the preparation of diagnostically functional silver SERS substrates. Compared to other structures, Ag dendrites possess many multilevel branching nanostructures, thus allowing a large specific surface area and the corresponding complex nanostructure may be more favorable to absorb probe molecules. A strong electromagnetic coupling can be formed in the space between two adjacent branches from the coupling of SPR. Thus, a large number of hotspots would exist in the spaces at the end of branches or among lateral $\mathrm{Ag}$ branches. These factors should favor $\mathrm{Ag}$ dendritic nanostructures to be used as high-active SERS substrates. By varying the number and duration of pulses in a double-potentiostatic electrodeposition of silver substrates, the dendritic stage of growth, density, and distribution of the resulting shape of the deposited silver structure could be controlled. The morphology of the surfaces was determined by direct SEM imaging (Fig. 1).

There is a clear increase in the surface coverage density with an increasing number of pulses and time of electrodeposition. The optimum composition of the electrolyte solution for deposition was found to be $0.01 \mathrm{~mol} \mathrm{dm}{ }^{-3} \mathrm{AgNO}_{3}$. This low concentration of $\mathrm{Ag}^{+}$ions causes the deposition of silver fractals of a dendritic shape with a microdimensional stem and nanosized lateral branches. It is assumed that the "hotspots" formed between the individual dendritic branches are responsible for signal enhancement. ${ }^{46}$

All stages of the dendritic growth were deposited on the ITO glass (Fig. 2). Nanosized nuclei with sizes from 200 to $900 \mathrm{~nm}$ were observed in the Ag substrates A, E, I, and M (Fig. 1) deposited with 10 pulses at all durations of $\mathrm{E}_{2}$. There was a noticeable lengthening of the stems and growth of lateral branches with increasing numbers of pulses at a constant duration of potential $E_{2}$. The length of the main stem increased from 800 to $6000 \mathrm{~nm}$ and the smallest lateral branches from 80 to $200 \mathrm{~nm}$. The density of the coating visibly increased with an increasing number of pulses and surfaces L, O, and P (Fig. 1) were completely covered by the silver dendritic structures in multiple threedimensional layers. Subsequent SERS analysis and calculation of signal enhancement factor (SEF) showed that the highest values of SEF were provided by surface D (Fig. 1). Too dense coverage of the ITO glass by dendrites was not suitable for SERS signal enhancement. On the other hand, a light coverage of ITO glass by silver structures was not SERS active when the distance between deposited dendrites was too large.

\subsection{Surface Hydrophobicity Test Measurements}

The measurement of the contact angle and surface hydrophobicity is important because of the need for the sample to be concentrated at the point of analysis. A sample spreading over the hydrophilic surface of the silver substrate leads to a decreased local concentration and a reduction in the number of detectable analyte molecules. Therefore, the preparation of a surface that is superhydrophobic should lead to more detailed and accurate measurement of the final SERS signal. The contact angle values for water increased for all the silver surfaces from those prepared with 10 pulses to 70 pulses (Table 1). All substrates were hydrophobic, and samples C, D, H, and P (Fig. 1) were evaluated as superhydrophobic because the contact angle was over $150 \mathrm{deg}$. For the nonpolar solvent thiodiglycol, the contact angles decreased from 10 pulses to 50 pulses and then increased at the surface prepared with 70 pulses. The irregular changes in 

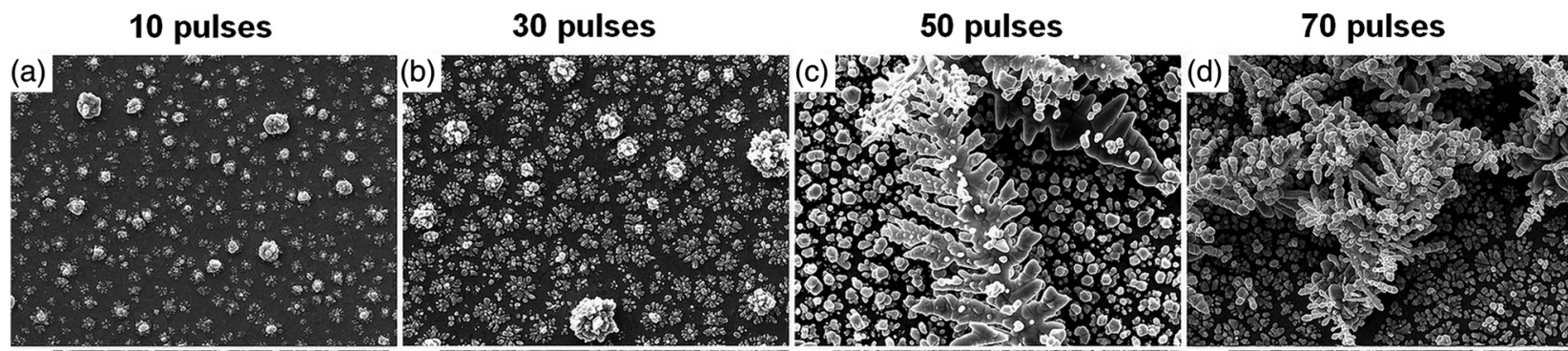

$0.5 \mathrm{~s}$
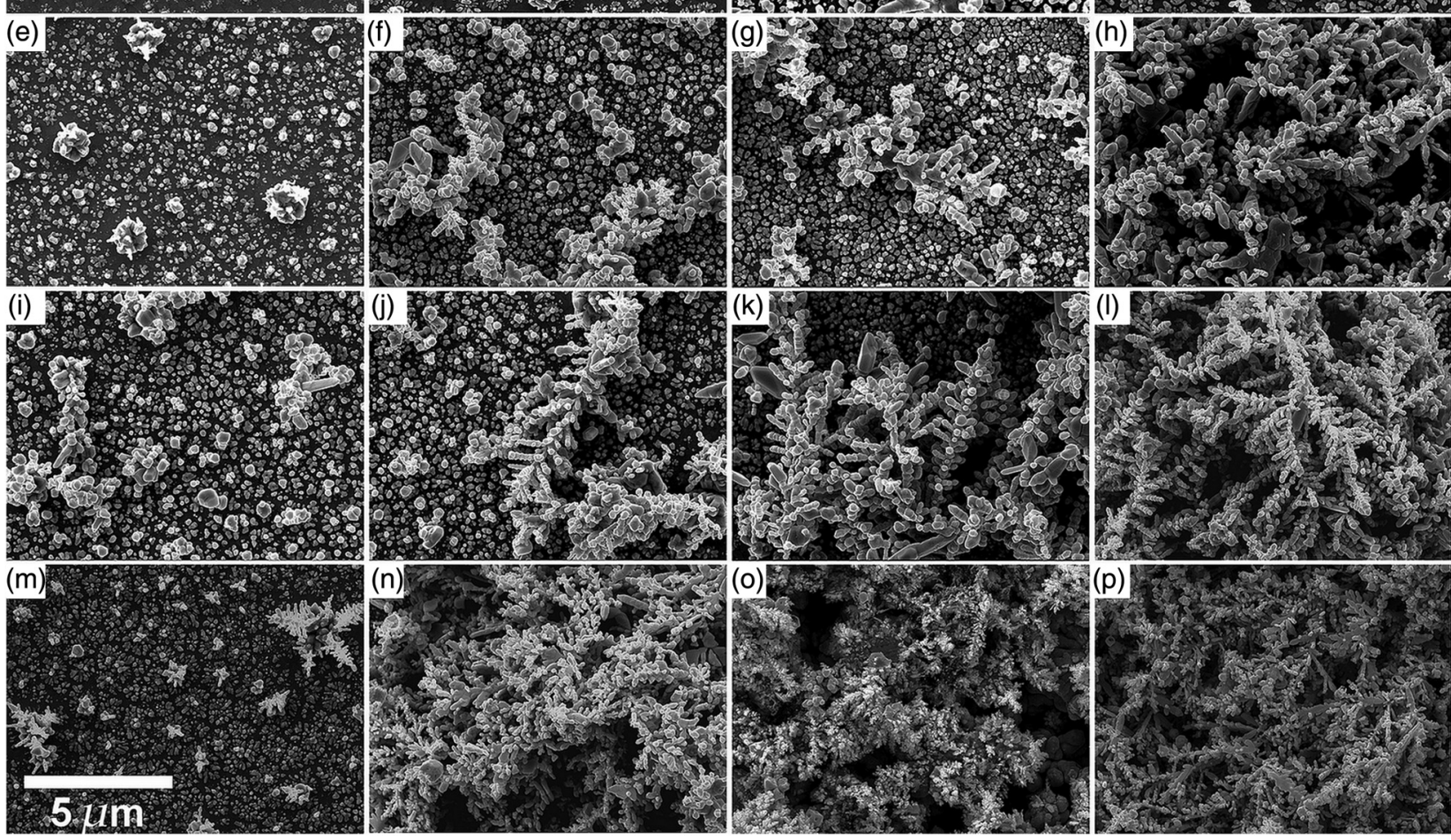

Fig. 1 SEM images of silver films with dendritic geometry; magnification 10,000x. Surfaces prepared by $10,30,50$ and 70 pulses (from the left to right) are in rows: $A \rightarrow D, E \rightarrow H, I \rightarrow L$, and $M \rightarrow P$. Surfaces prepared by various $E_{2}$ potential durations $(0.5,1.0,1.5$ and $2.0 \mathrm{~s})$ are in columns from top to bottom: $\mathrm{A} \rightarrow \mathrm{M}, \mathrm{B} \rightarrow \mathrm{N}, \mathrm{C} \rightarrow \mathrm{O}$, and $\mathrm{D} \rightarrow \mathrm{P}$.

the contact angle of thiodiglycol were probably due to its low surface tension. The trend for glycerol as a polar solvent was opposite to that of water, and the contact angles decreased from 10 pulses to 70 pulses.

A dependence of the SERS signal enhancement of R6G with the contact angle was observed. Figure 3 shows an increase of the signal enhancement with increasing the contact angle values for a peak with Raman shift of $770 \mathrm{~cm}^{-1}$ [Fig. 3(a)] and $1360 \mathrm{~cm}^{-1}$ [Fig. 3(b)]. The silver surfaces A, E, I, and M, with the lowest values of contact angle, and the sparse coverage of the silver dendritic structures showed the lowest signal values for both SERS peaks. This was thought to be because the distance between each nanostructure was too large to create an electromagnetic field strong enough to provide signal enhancement. By contrast, the hydrophobic surfaces $\mathrm{D}, \mathrm{H}, \mathrm{L}$, and $\mathrm{P}$ with contact angles about $150 \mathrm{deg}$ showed significant enhancement.

\subsection{Results of the Electromagnetic Simulation of Ag Surface}

Figure 4 shows the FDTD simulation of the electric field on a dendritic nanostructure. The hotspots on the edge of the branches, where the enhancement of the electric field is maximal, are clearly visible. Additional hotspots are present on the folds, where the spherical nanoparticles are linked to each other and to the trunk.

\subsection{SERS Analysis of Rhodamine 6G}

The SERS spectra of R6G with different concentrations were recorded using the silver dendritic substrates (A-P) in the Raman shift region from 400 to $1800 \mathrm{~cm}^{-1}$. A representative Raman spectrum with concentration $1 \times 10^{-6} \mathrm{~mol} \mathrm{dm}^{-3}$ on substrate $\mathrm{D}$ is presented in Fig. 5 and the corresponding vibrational modes are shown in Table 2.

To probe the detection limit of the silver surface $D$, R6G with concentrations from $1 \times 10^{-6}$ to $1 \times 10^{-12} \mathrm{~mol} \mathrm{dm}^{-3}$ were compared (Fig. 6).

The characteristic peaks of R6G are clearly identified in all spectra, and the peak intensity increased with increasing R6G concentration (Fig. 6) over the range from $1 \times 10^{-5}$ to $1 \times$ $10^{-12} \mathrm{~mol} \mathrm{dm}^{-3}$ (Fig. 7). SERS spectra were measured and averaged from 10 random points on the surface. We selected two representative peaks: $770 \mathrm{~cm}^{-1}$ corresponds to $\mathrm{C}-\mathrm{CH}$ out of plane bend mode and $1360 \mathrm{~cm}^{-1}$ corresponds to $\mathrm{C}-\mathrm{C}$ aromatic stretching vibrations, for analysis of the concentration-dependent 


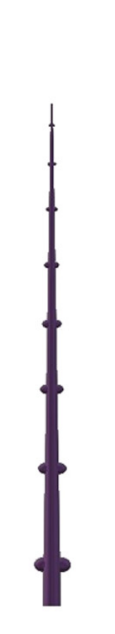

nucleus I. stage

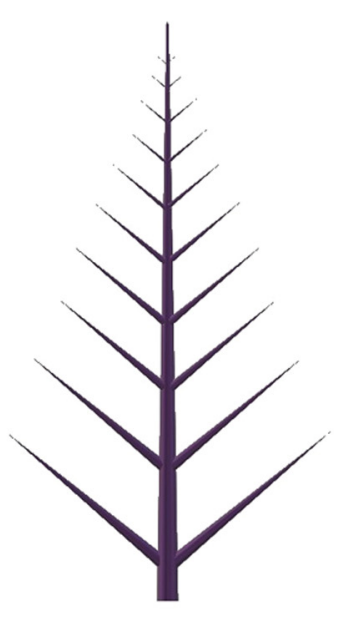

II. stage

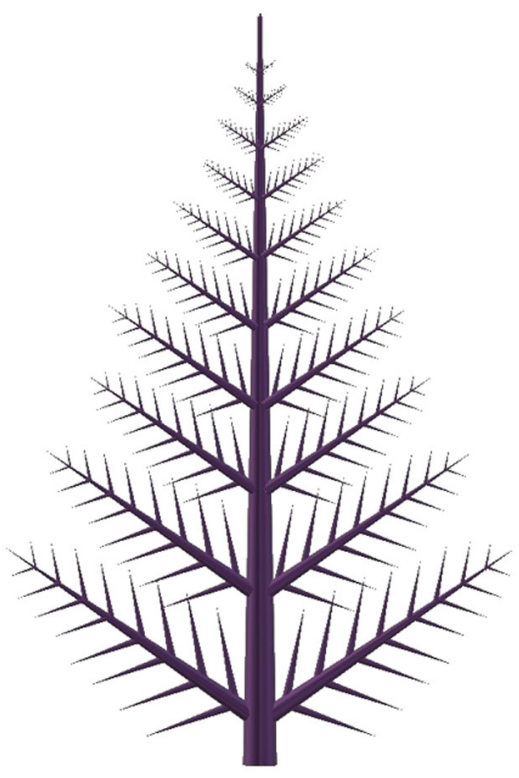

III. stage

Fig. 2 Stages of growth of dendritic structures. There are initially nanosized Ag nuclei, which in the first stage of growth form the supporting stem. The silver dendrite in the second stage has formed lateral branches, which in the final phase grow up to additional lateral branches.
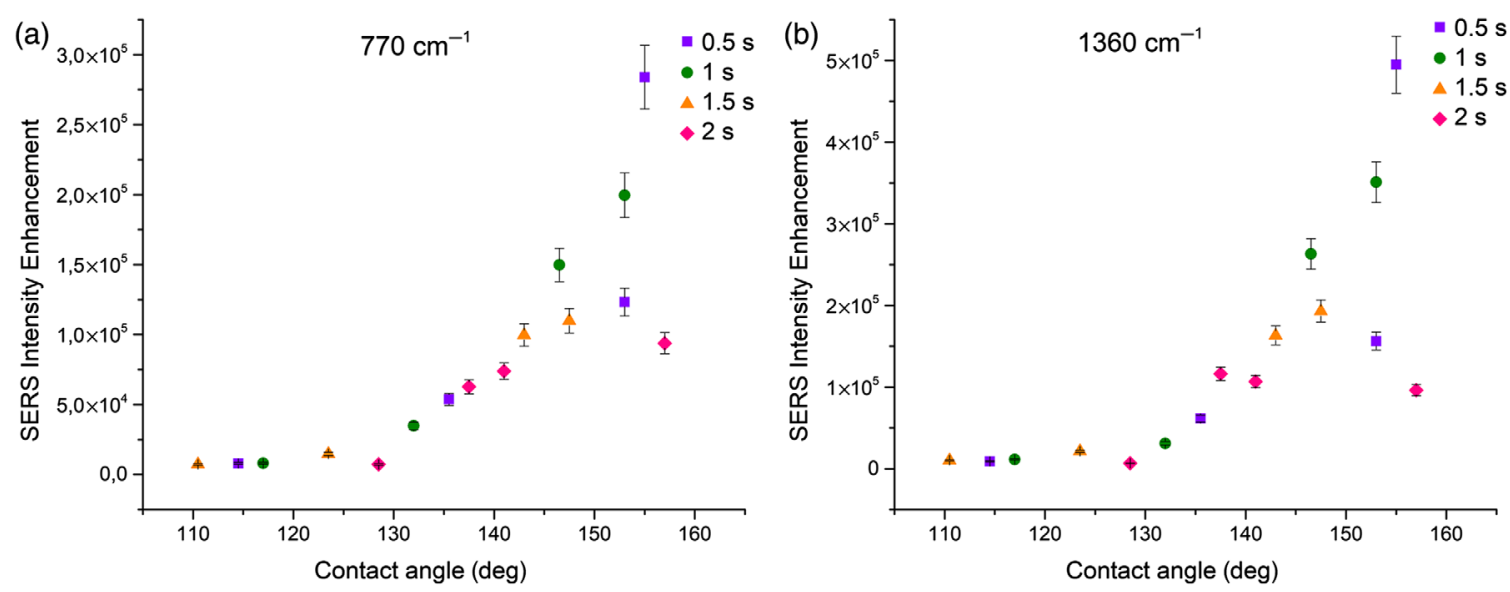

Fig. 3 Dependence of SERS signal enhancement with the contact angle at different pulse rates for R6G. (a) Raman intensity of the peak at $770 \mathrm{~cm}^{-1}$ as a function of contact angle. (b) Raman intensity of the peak at $1360 \mathrm{~cm}^{-1}$ as a function of contact angle.

variation. The signals evidently decrease gradually, however, nonlinearly, with decreasing concentration of R6G. The deviation of the peak intensity at $770 \mathrm{~cm}^{-1}$ was calculated as $17.1 \%$ and for peak $1360 \mathrm{~cm}^{-1}$ as $15.9 \%$.

The SEF is an important indicator of the SERS activity of a substrate. The analytical SEF of the silver SERS substrates was calculated using Eq. (1): ${ }^{47}$

$\mathrm{SEF}=\frac{I_{\mathrm{SERS}} \cdot C_{\mathrm{ref}}}{I_{\mathrm{ref}} \cdot C_{\mathrm{SERS}}}$,

where $C_{\text {ref }}$ and $C_{\text {SERS }}$ are the reference concentration and sample concentration, respectively, and $I_{\text {ref }}$ and $I_{\text {SERS }}$ are the signal intensity of the Raman peak, in this case, at $1360 \mathrm{~cm}^{-1}$ (the most intense peak in the spectrum). The enhancement factor was determined as $2.01 \times 10^{9}$ using $1 \times 10^{-3} \mathrm{~mol} \mathrm{dm}^{-3} \mathrm{R} 6 \mathrm{G}$ on a reference surface of $100 \mathrm{~nm} \mathrm{Ag}$ sputtered on silicon and $1 \times 10^{-12} \mathrm{~mol} \mathrm{dm}^{-3}$ R6G measured on silver SERS substrate D, which was then selected for further study.

\subsection{SERS Analysis of Blood Plasma}

As it gave the strongest enhancement, the silver SERS substrate $\mathrm{D}$ was integrated into a plastic cassette to provide a compact diagnostic platform for direct use in the medical lab (Fig. 8).

Using this equipment, the main peaks in human blood plasma, diluted 1:9 with water, could be detected by SERS analysis with high signal intensity (Fig. 9).

The spectrum represents the vibrational modes of various biomolecules, such as proteins, lipids, and nucleic acids, which could change in quantity or conformation with the 


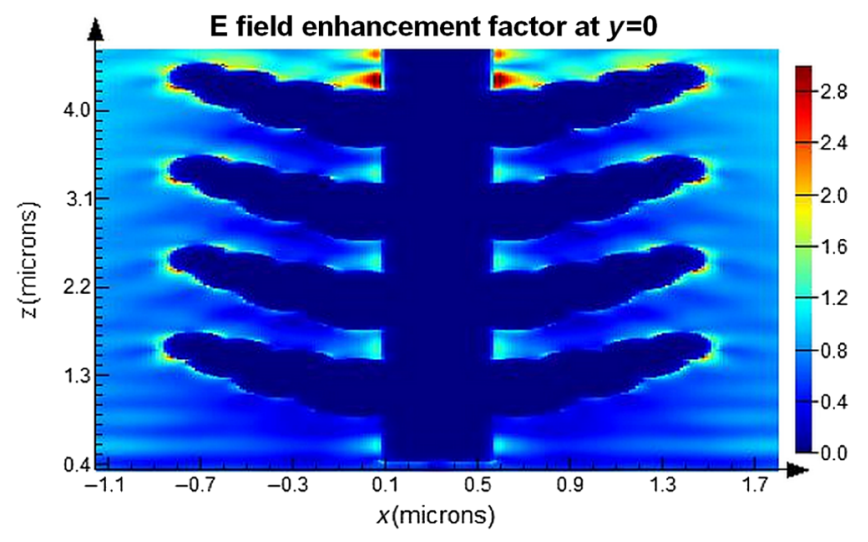

Fig. 4 FDTD simulation of electric field distribution on silver dendritic structure under 532-nm laser excitation. The hot spots are visible on the edge of the branches and on the folds, where the enhancement of the electric field reaches maximum values.

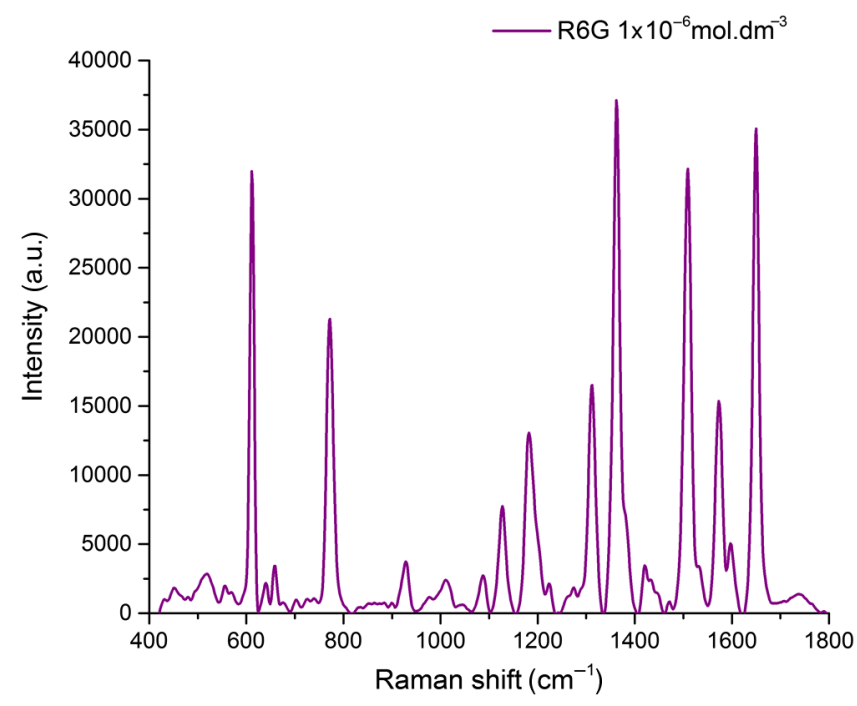

Fig. 5 Representative spectra of $1 \times 10^{-6} \mathrm{~mol} \mathrm{dm}^{-3} \mathrm{R} 6 \mathrm{G}$ on SERS substrate $D$ with subtracted background recorded with an excitation wavelength at $532 \mathrm{~nm}$.

Table 2 The characteristic Raman shift of the most intense peaks observed in the SERS spectrum of R6G recorded at $\lambda_{\mathrm{exc}}=532 \mathrm{~nm}$.

\begin{tabular}{ll} 
Peak position $\left(\mathrm{cm}^{-1}\right)^{\mathrm{a}}$ & \multicolumn{1}{c}{ Vibrational mode } \\
\hline $612 \mathrm{vs}$ & $\mathrm{C}-\mathrm{C}-\mathrm{C}$ ring in-plane \\
$770 \mathrm{~s}$ & $\mathrm{C}-\mathrm{H}$ out of plane bend \\
$1127 \mathrm{~m}$ & $\mathrm{C}-\mathrm{H}$ in-plane bend \\
$1184 \mathrm{~m}$ & $\mathrm{C}-\mathrm{H}$ in-plane bend \\
$1313 \mathrm{~m}$ & $\mathrm{~N}-\mathrm{H}$ in-plane bend \\
$1360 \mathrm{vs}$ & $\mathrm{C}-\mathrm{C}$ aromatic stretching \\
$1509 \mathrm{vs}$ & $\mathrm{C}-\mathrm{C}$ aromatic stretching \\
$1574 \mathrm{~m}$ & $\mathrm{~N}-\mathrm{H}$ in-plane bend \\
$1650 \mathrm{vs}$ & $\mathrm{C}-\mathrm{C}$ aromatic stretching \\
\hline
\end{tabular}

${ }^{\mathrm{a}} \mathrm{m}$, medium; s, strong; vs, very strong.

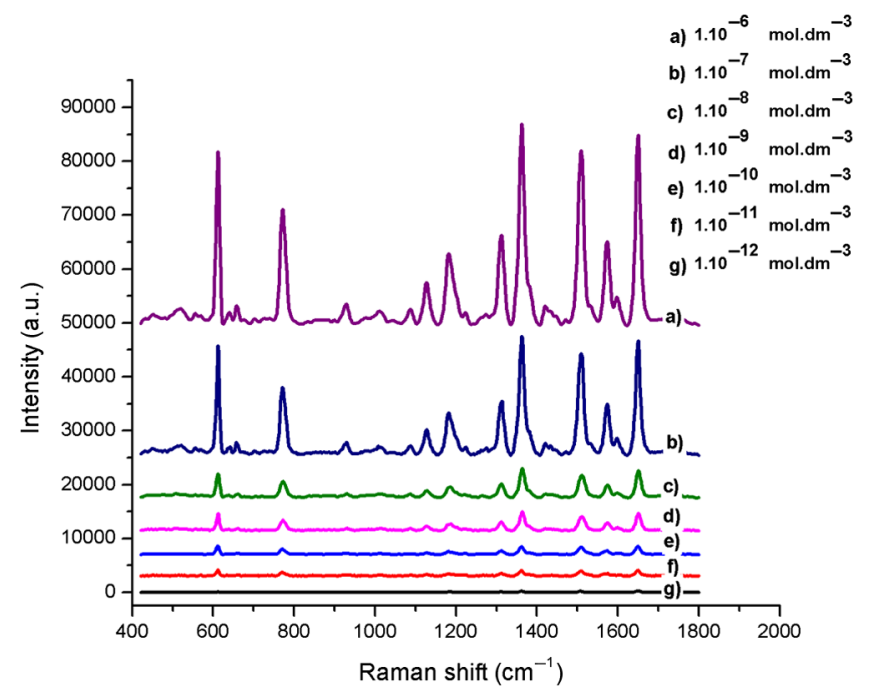

Fig. 6 SERS spectra of R6G on silver surface $D$ at concentrations from $1 \times 10^{-6} \mathrm{~mol} \mathrm{dm}^{-3}$ to $1 \times 10^{-12} \mathrm{~mol} \mathrm{dm}^{-3}$ with subtracted background recorded with an excitation wavelength at $532 \mathrm{~nm}$.

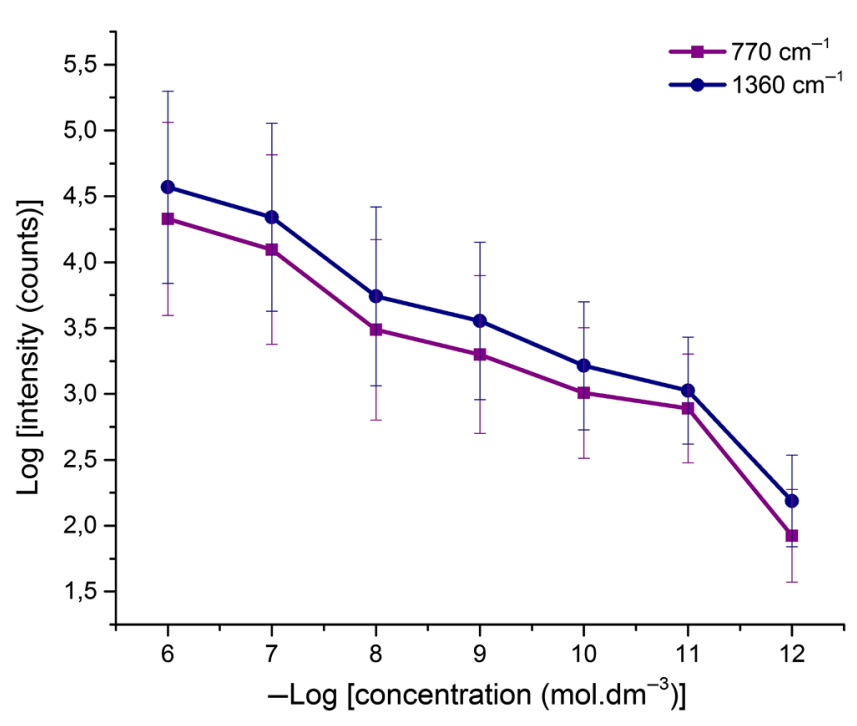

Fig. 7 A negative logarithmic plot of R6G concentration versus logarithmic signal intensity for the peak at 770 and $1360 \mathrm{~cm}^{-1}$. The deviation of the peak intensity at $770 \mathrm{~cm}^{-1}$ was calculated as $17.1 \%$ and for peak $1360 \mathrm{~cm}^{-1}$ as $15.9 \%$.

development of colorectal cancer. Tentative assignments for the main peaks are listed in Table $3 .^{31}$

When comparing the results from healthy patients with those with cancer, determining sensitivity and selectivity is a necessary part of the evaluation of clinical research results. A high sensitivity [Eq. (2)] is a required characteristic for initial diagnostic tests and measures the ability of the test to correctly identify all those patients with the disease. Further specific and detailed tests may show that some of the patients do not have the disease and these are then identified as false positives. By contrast, the specificity of a clinical trial [Eq. (3)] refers to the ability of the test to identify those patients without the disease correctly. The combination of an initial test with high sensitivity/low specificity and a second test with low sensitivity/ high specificity should produce a diagnostic method, which 


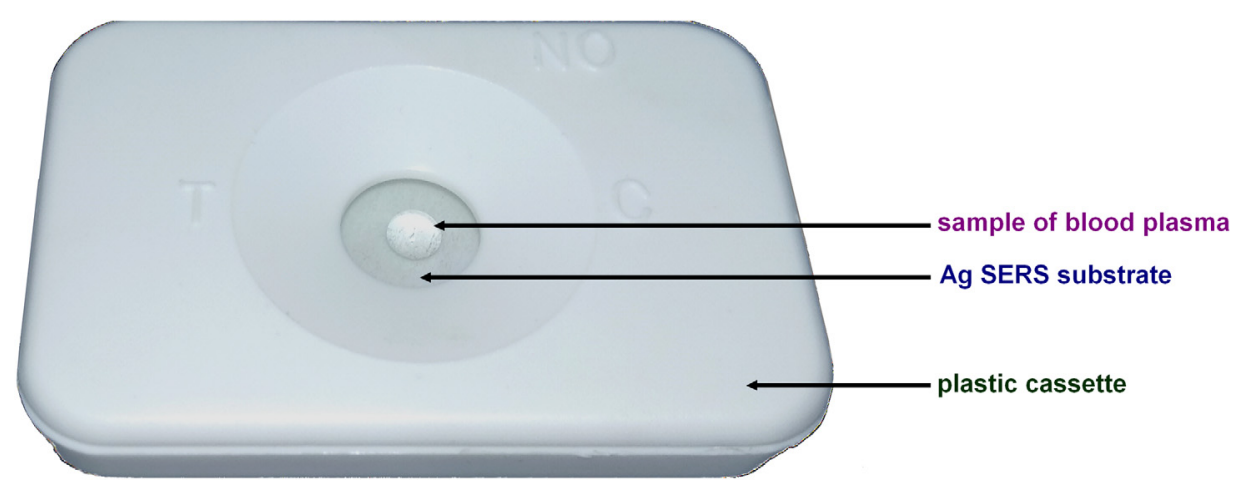

Fig.8 Plastic cassette with integrated silver SERS substrate as a diagnostic platform for direct use in medical lab. It provides a protective function and improves the handling of the SERS diagnostic substrate.

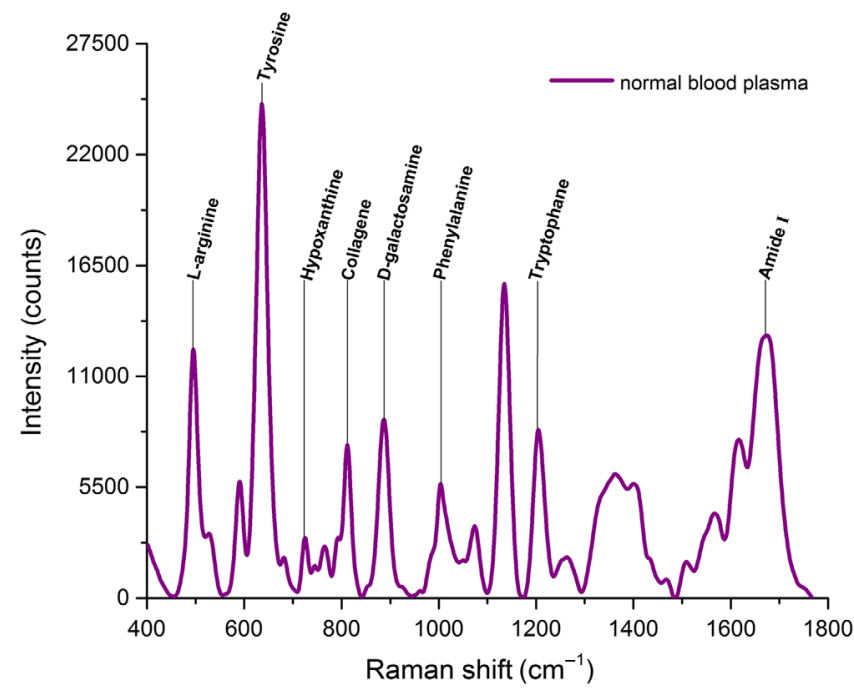

Fig. 9 SERS spectrum of human blood plasma diluted $1: 9$ with water on electrodeposited silver substrate with subtracted background recorded with an excitation wavelength at $725 \mathrm{~nm}$.

will correctly find all the true positives and then correctly identify any false positives as actually negatives: ${ }^{48}$

Sensitivity $=\frac{\text { True positives }}{\text { True positives }+ \text { False negatives }}$,

Specificity $=\frac{\text { True negatives }}{\text { True negatives }+ \text { False positives }}$.

The SERS of blood plasma samples were recorded from 15 healthy volunteers (normal samples) and 15 colorectal cancer patients. The measured spectra were clean, with no interfering signals in the spectral range of interest. All the measured SERS spectra were normalized to the integrated area under the curve from 400 to $1800 \mathrm{~cm}^{-1}$ and the mean spectra with their standard deviations were overlaid [Fig. 10(a)].

All the main SERS peaks (Table 3) were observed in both normal and cancer samples. The SERS peaks at 725 and $881 \mathrm{~cm}^{-1}$ were more intense in cancer samples, in comparison to the normal samples [Fig. 10(a), lower trace]. All other measured SERS peaks were more intense for the normal samples. The resulting difference map of the variations of the major peaks is shown in Fig. 10(b).
Table 3 Peak assignment of the characteristic Raman shift observed in the SERS spectrum of blood plasma recorded at $\lambda_{\text {exc }}=785 \mathrm{~nm}^{31}$

\begin{tabular}{lcc} 
Peak position $\left(\mathrm{cm}^{-1}\right)^{\mathrm{a}}$ & Vibrational mode $^{\mathrm{b}}$ & Major assignment \\
\hline $494 \mathrm{~s}$ & $\nu(\mathrm{S}-\mathrm{S})$ & L-arginine \\
$589 \mathrm{w}$, sh & & Amide-VI \\
$638 \mathrm{vs}$ & $\nu(\mathrm{C}-\mathrm{S})$ & Tyrosine \\
$725 \mathrm{w}$ & $\delta(\mathrm{C}-\mathrm{H})$ & Adenine \\
$818 \mathrm{w}$ & $\nu(\mathrm{C}-\mathrm{C})$ & Collagen \\
$823 \mathrm{~m}$ & Ring breathing & Tyrosine \\
$881 \mathrm{~m}$ & $\delta($ ring $)$ & Tryptophan \\
$1004 \mathrm{~m}$ & $\nu_{s}(\mathrm{C}-\mathrm{C})$ & Phenylalanine \\
$1074 \mathrm{w}$ & $\nu(\mathrm{C}-\mathrm{C})$ & Phospholipids \\
$1131 \mathrm{~s}$ & $\nu(\mathrm{C}-\mathrm{C})$ & D-Mannose \\
$1206 \mathrm{~m}$ & Ring vibration $_{1322 \mathrm{~m}, \text { sh }}$ & Tyrosine \\
$1360 \mathrm{~m}, \mathrm{br}$ & $\mathrm{CH}_{3} \mathrm{CH} \mathrm{H}_{2}$ twisting & Collagen, tryptophan \\
$1402 \mathrm{~m}$, sh & & Tryptophan \\
$1573 \mathrm{w}$ & $\nu(\mathrm{C}-\mathrm{C})$ & Phospholipids \\
$1655 \mathrm{~s}$ & $\nu\left(\mathrm{C}-\mathrm{C}_{6} \mathrm{H}_{5}\right)$ & DNA/RNA bases \\
\hline
\end{tabular}

${ }^{\mathrm{a}} \mathrm{vw}$, very weak; w, weak; $\mathrm{m}$, medium; s, strong; vs, very strong; sh, shoulder; and br, broad.

${ }^{\mathrm{b}} \nu$, stretching; $\nu \mathrm{S}$, symmetric stretching; and $\delta$, in-plane deformation.

To compare the spectra from oncology patients to normal subjects, a comparative analysis based on the mean intensities and relative standard deviations was performed. These most significant deviations were observed in the Raman peaks at 494, 638, 725, 823, 881, 1206, and $1655 \mathrm{~cm}^{-1}$ [Fig. 10(b)]. Two SERS peaks, whose intensities obviously differ between normal and cancer blood plasma samples, at $725 \mathrm{~cm}^{-1}$, assigned to adenine, and $638 \mathrm{~cm}^{-1}$, to tyrosine, were considered important differential diagnostic parameters. 

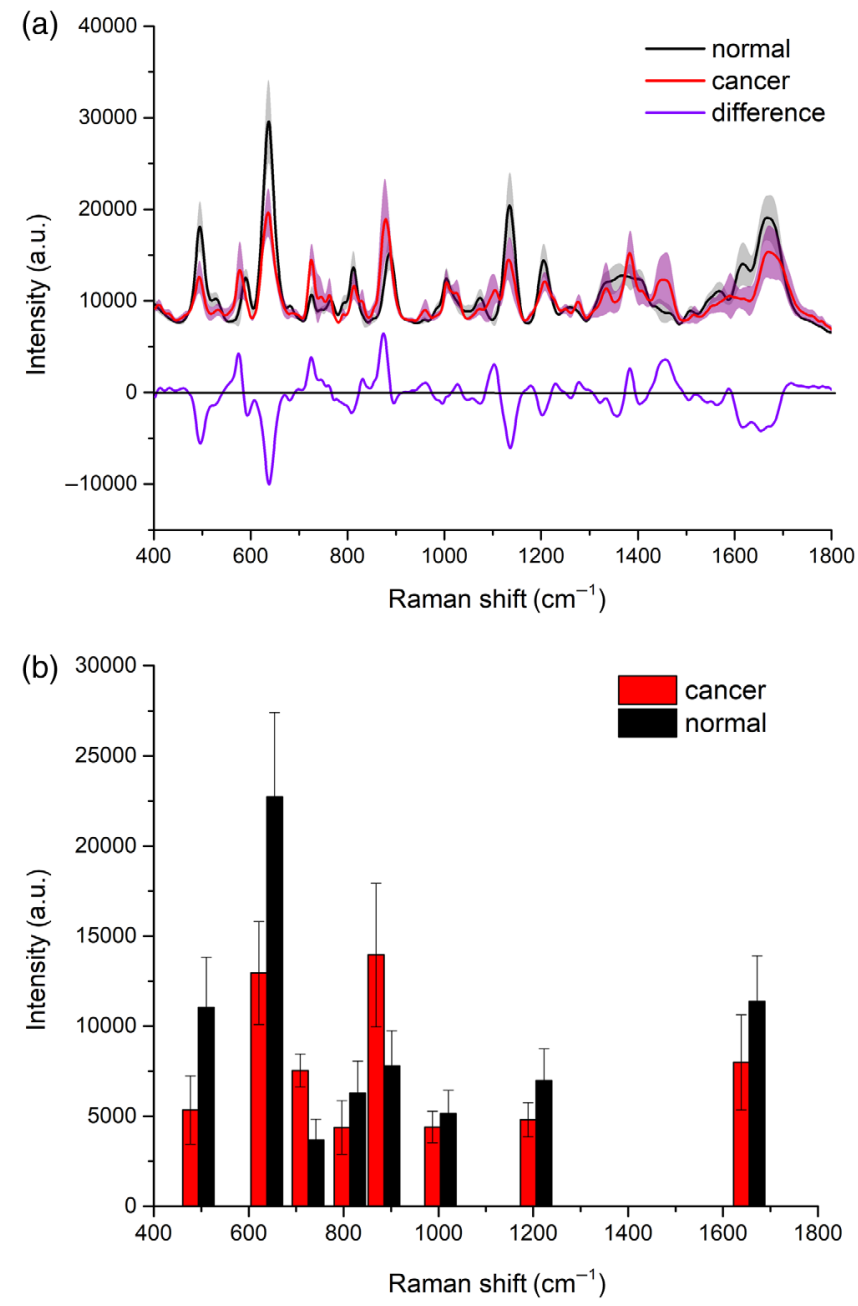

Fig. 10 (a) Comparison of the mean spectra for cancer patient samples (red curve, $n=15$ ) and mean spectra of the normal patient samples (black curve, $n=15$ ). The shaded areas represent the standard deviations of the means. The difference spectrum (at the bottom) represents the differences between the means of the two groups (cancer/normal). (b) Comparison of the mean intensities and standard deviations of selected peaks of samples of cancer (red column, $n=15$ ) patient and normal (black column, $n=15$ ) patient blood plasma.

\subsection{Result of Statistical Analysis}

The ratios of the intensities of selected pairs of peaks for individual samples were compared (Fig. 11).

The mean value (mean $\pm \mathrm{SD}$ ) of the $I_{725}$ versus $I_{638}$ ratio (adenine peaks compared to tyrosine) for cancer plasma samples $(0.58 \pm 0.15, n=15)$ was significantly different from the mean ratio for normal plasma samples $(0.18 \pm 0.04, n=15)$. Further examination of the ratios of the peak intensity at $725 \mathrm{~cm}^{-1}$ (adenine) to the peak intensities at $494 \mathrm{~cm}^{-1}$ (L-arginine) and $1655 \mathrm{~cm}^{-1}$ (amide I peak of proteins) gave the mean values (mean $\pm \mathrm{SD}$ ) for the ratios of $I_{725} / I_{494}$ and $I_{725} / I_{1655}$ for normal plasma samples of $0.32 \pm 0.06$ and $0.16 \pm 0.04$ and for cancer plasma samples of $1.77 \pm 0.84$ and $0.60 \pm 0.12$, respectively. The separation of the samples can be represented by decision lines calculated from average values of compared intensities of normal and cancer samples, where $(\mathrm{A})=0.38$, $(B)=1.02$, and $(C)=0.67$ (Fig. 11). When the ratios were compared using the student's $t$-test $(p<0.05)$, the values for the colorectal cancer and normal plasma samples were statistically and significantly different. From these ratios, the sensitivity of the test was calculated as $I_{725} / I_{638}=94 \%, I_{725} / I_{494}=87 \%$, and $I_{725} / I_{1655}=87 \%$, respectively. Further examination found that the $I_{725} / I_{638}$ ratio for all the plasma samples from the normal group was identified with $100 \%$ specificity so that the test using this ratio satisfied both the sensitivity and specificity criteria.

For a more comprehensive comparison, $\mathrm{PCA}^{49}$ of the intensities at the wavelengths of the selected peaks from Fig. 10(b) was carried out, which reduces the primary variance in the original dataset to a few principal component (PCs) variables. These PCs can be used to build a model with a resolution of recognition. ${ }^{49}$ The fluorescence background of the original SERS data was first removed using a modified multipolynomial fitting algorithm. All spectra were normalized by the integrated area under the curve and then dataset was fed into the XLSTAT software for PCA analysis. The PC1 accounted for $69 \%$ of the variance, $\mathrm{PC} 2$ for $14 \%$, and $\mathrm{PC} 3$ for $8 \%$.

Comparisons of the scores for PC1 and PC2 [Fig. 12(a)] show two separate groups of colorectal cancer data (red points) and normal plasma points (black points). Samples from the two groups were separated from each other with $100 \%$ sensitivity and specificity. Two differentiated groups of points were also observed when combining PC1 and PC3 [Fig. 12(b)]. This
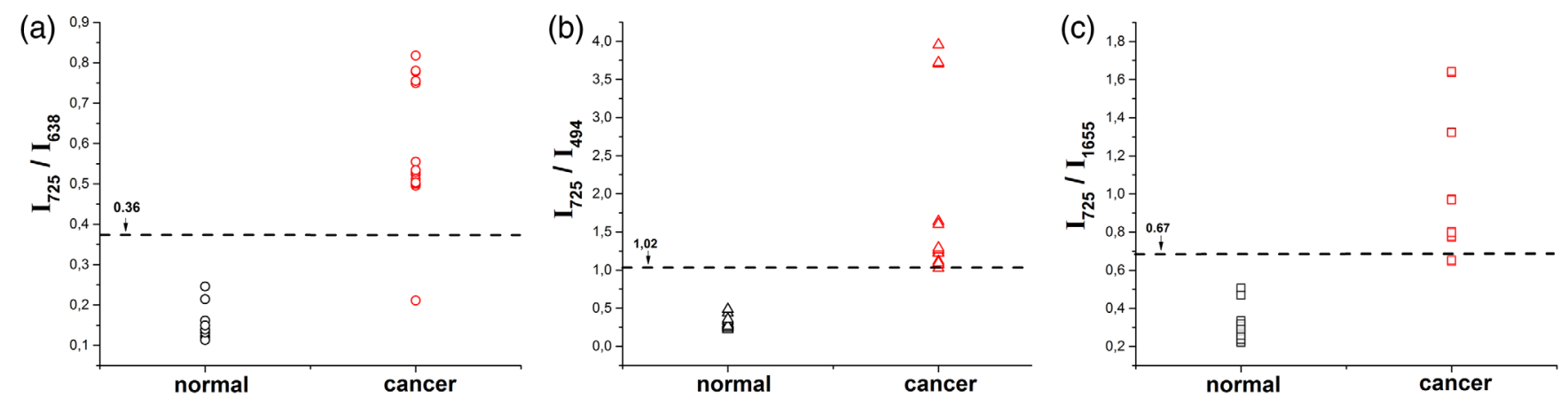

Fig. 11 Scatter plot of the intensity ratio of the Raman signal for (a) 725 versus $638 \mathrm{~cm}^{-1}$, (b) 725 versus $494 \mathrm{~cm}^{-1}$, and (c) 725 versus $1655 \mathrm{~cm}^{-1}$. The values of dotted decision line were calculated from average values of compared intensities of normal and cancer samples, where $(a)=0.38,(b)=1.02$, and (c) $=0.67$. 

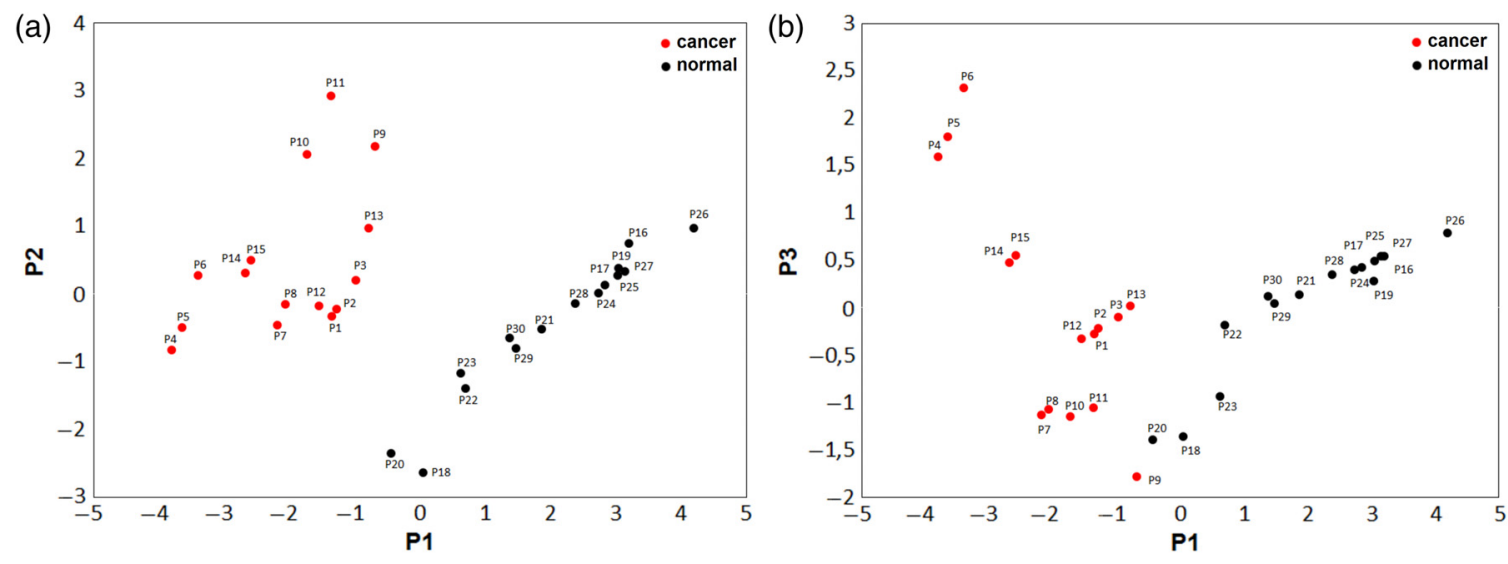

Fig.12 (a) Plots of the PC1 versus the PC2 for normal group (black points) and cancer group (red points). The two groups were separated with $100 \%$ sensitivity and specificity. (b) Plot of the PC1 versus PC3 for normal group (black points) and cancer group (red points). The two groups were separated with $94 \%$ sensitivity and $100 \%$ specificity.

option was found to be capable to distinguish cancer samples from normal samples with $94 \%$ sensitivity and $100 \%$ specificity.

\section{Discussion}

This initial clinical study demonstrated statistically significant differences between the blood plasma of oncology patients and healthy volunteers, which have been successfully detected by SERS. Colloidal solutions of nanoparticles of noble metals used in previous research of Feng and Lin were replaced by electrochemically deposited silver dendritic SERS substrates. ${ }^{7,31}$ Ag dendrites possess multilevel branching nanostructures with a large specific surface area with a large number of hotspots at the edge of branches or among adjacent $\mathrm{Ag}$ branches. These Ag dendritic nanostructures with proven high SEF can be used as a high-active SERS substrates for detection of changes in blood plasma composition.

It is proposed that the intensity differences between cancer and normal serum are the results of molecular changes associated with carcinogenesis. The SERS peak at $1655 \mathrm{~cm}^{-1}$ is attributed to the amide I peak of proteins in the $\alpha$-helix conformation and human serum albumin as a principal extracellular transport protein. This protein is a globular molecule with 17 disulfide bridges, which is also observed as a SERS peak for S-S stretching vibration at $494 \mathrm{~cm}^{-1} .{ }^{50}$ In cancer serum, both of these two SERS peaks are less intensive, indicating that colorectal cancer may be associated with a decrease in the relative amounts of protein. Similar changes were observed in earlier studies of colorectal, nasopharyngeal, and gastric cancer blood plasma with colloidal SERS substrates. ${ }^{7,29,31}$ The peak at $725 \mathrm{~cm}^{-1}$ assigned to the $\mathrm{C}-\mathrm{H}$ bending mode of adenine was reported to be an important molecular selector for colorectal cancer diagnosis. ${ }^{31}$ Higher relative intensities of adenine in cancer serum than in normal serum were attributed to the cancerous metabolism of DNA or RNA bases in the serum of oncological patients. This appearance was explained by apoptosis and necrosis, or by the release of intact cells into the bloodstream and their subsequent lysis. ${ }^{51}$ The lower signal intensities of SERS peaks of tyrosine $\left(638,823\right.$, and $\left.1206 \mathrm{~cm}^{-1}\right)$ and L-arginine $\left(494 \mathrm{~cm}^{-1}\right)$ were observed in cancer blood serum than those of normal serum. These changes have been attributed to the abnormal metabolism of the tumor. ${ }^{29}$
To classify colorectal cancer and normal plasma samples, an efficient algorithm based on empirical analysis of SERS spectra in terms of peak intensity ratio measurements was used. The ratio of peaks at $725 \mathrm{~cm}^{-1}$ (adenine) and $638 \mathrm{~cm}^{-1}$ (tyrosine) was used in the study of Lin et al. ${ }^{31}$ It was found that the ratio of intensity of adenine to intensity of tyrosine was markedly higher in cancer plasma than in normal serum samples, and the results of empirical analysis of SERS spectra showed $68.4 \%$ sensitivity and $95.6 \%$ specificity. ${ }^{31}$ Intensity variations of ratio of $I_{725}$ versus $I_{638}$ are significantly noticeable in the cancer group, which agreed with Han's research. ${ }^{52}$ Differences between normal and colorectal cancer plasma may reflect changes in the relative amounts of potential biologic markers, according to the tentative assignments of plasma SERS peaks. Nonparametric intensity ratios may potentially be employed as an effective diagnostic algorithms for colorectal cancer detection and, in the present study, the multivariate statistical method PCA, which utilizes the entire spectra, was used due to the complexity of blood plasma composition. This analysis was performed to condense the recorded data to the important PCs. Scores of PC1 versus PC2 and PC1 versus PC3 for the normal and colorectal cancer sample spectra were found to distinguish the two groups. The diagnostic $97.4 \%$ sensitivity and $100 \%$ specificity was achieved. ${ }^{31}$

\section{Conclusion}

Preliminary experiments have suggested a diagnostic test for the early detection of colorectal cancer by SERS analysis of human blood plasma, which can be used in clinical practice. Easily made, low-cost, and rapidly deposited electrochemically prepared silver surfaces can be used as SERS substrates. The pulsed deposited silver surface created with 70 pulses with $E_{2}=$ $-0.1 \mathrm{~V}$ for $0.5 \mathrm{~s}$ had the highest enhancement factor $\left(2.1 \times 10^{9}\right)$ for R6G. This SERS surface was superhydrophobic, which allowed the sample to be preconcentrated at a fixed place on the substrate and prevent unnecessary spreading and flowing of the sample over the surface.

Comparison of the SERS spectra on this surface from 15 colorectal cancer patients and 15 healthy volunteers enabled the two groups to be differentiated by comparing the intensity $I_{725} / I_{638}$ with the $100 \%$ specificity and $94 \%$ sensitivity. PCA analysis of the SERS spectra found that the diagnostics was 
performed with $100 \%$ sensitivity and specificity for PC1 versus PC2 and 94\% sensitivity and 100\% specificity for PC1 versus PC3. These promising results now need to be confirmed on a larger sample of subjects to generate a more accurate and reliable diagnostic model, which could be suitable to apply in medical practice, thereby enabling early, rapid, and noninvasive diagnostics of colorectal cancer.

\section{Disclosures}

The authors have no relevant financial interests in this article and no potential conflicts of interest to disclose.

\section{Acknowledgments}

This research has been financially supported by grant VEGA 1/ 0074/17 of the Slovak Scientific Grant Agency and APVV-160029 of the Slovak Research and Development Agency. Clinical research was approved by the Ethics committee of Louis Pasteur University Hospital in Kosice.

\section{References}

1. M. Arnold et al., "Global patterns and trends in colorectal cancer incidence and mortality," Gut 66(4), 683-691 (2017).

2. D. K. Rex et al., "Colorectal cancer screening: recommendations for physicians and patients from the U.S. multi-society task force on colorectal cancer," Am. J. Gastroenterol. 112(7), 1016-1030 (2017).

3. T. K. Burki, "Flexible sigmoidoscopy screening for colorectal cancer," Lancet Oncol. 15(10), e425 (2014).

4. A. Phalguni et al., "Tests detecting biomarkers for screening of colorectal cancer: what is on the horizon?" GMS Health Technol. Assess. 11, Doc01 (2015).

5. C. H. Chen, C. P. Wen, and M. K. Tsai, "Fecal immunochemical test for colorectal cancer from a prospective cohort with 513, 283 individuals," Medicine (Baltimore) 95(36), e4414 (2016).

6. L. M. Lobigs et al., "The use of biomarkers to describe plasma-, red cell- and blood volume from a simple blood test," Am. J. Hematol. 92(1), 62-67 (2017).

7. S. Feng et al., "Gastric cancer detection based on blood plasma surfaceenhanced Raman spectroscopy excited by polarized laser light," Biosens. Bioelectron. 26(7), 3167-3174 (2011).

8. X. Gao et al., "Paper-based surface-enhanced raman scattering lateral flow strip for detection of neuron-specific enolase in blood plasma," Anal. Chem. 89(18), 10104-10110 (2017).

9. K. Lin, D. L. P. Cheng, and Z. Huang, "Optical diagnosis of laryngeal cancer using high wavenumber Raman spectroscopy," Biosens. Bioelectron. 35(1), 213-217 (2012).

10. L. C. Ming et al., "Real time near-infrared Raman spectroscopy for the diagnosis of nasopharyngeal cancer,' Oncotarget 8(30), 49443-49450 (2017).

11. P. Gao et al., "Review article the clinical application of raman spectroscopy for breast cancer detection," J. Spectrosc. 2017, 1-10 (2017).

12. H. C. McGregor et al., "Real-time endoscopic Raman spectroscopy for in vivo early lung cancer detection," J. Biophotonics 10(1), 98-110 (2017).

13. A. Daniel, A. Prakasarao, and S. Ganesan, "Near-infrared Raman spectroscopy for estimating biochemical changes associated with different pathological conditions of cervix," Spectrochim. Acta A Mol. Biomol. Spectrosc. 190, 409-416 (2018).

14. P. Crow et al., "Assessment of fiberoptic near-infrared raman spectroscopy for diagnosis of bladder and prostate cancer," Urology 65(6), 1126-1130 (2005).

15. S. Mishra et al., "Recent advances in salivary cancer diagnostics enabled by biosensors and bioelectronics," Biosens. Bioelectron. 81, 181-197 (2016).

16. J. Zhao et al., "Using Raman spectroscopy to detect and diagnose skin cancer in vivo," Dermatol. Clin. 35(4), 495-504 (2017).

17. M. Jermyn et al., "Raman spectroscopy detects invasive brain cancer cells in humans," Biomed. Opt. Express 7(12), 5129-5137 (2016).
18. R. M. Jarvis and R. Goodacre, "Ultra-violet resonance Raman spectroscopy for the rapid discrimination of urinary tract infection bacteria," FEMS Microbiol. Lett. 232(2), 127-132 (2004).

19. G. V. Nogueira et al., "Raman spectroscopy study of atherosclerosis in human carotid artery," J. Biomed. Opt. 10(3), 031117 (2005).

20. J. A. M. Bispo et al., "Correlating the amount of urea, creatinine, and glucose in urine from patients with diabetes mellitus and hypertension with the risk of developing renal lesions by means of Raman spectroscopy and principal component analysis," J. Biomed. Opt. 18(8), 087004 (2013).

21. E. Ryzhikova et al., "Raman spectroscopy of blood serum for Alzheimer's disease diagnostics: Specificity relative to other types of dementia," J. Biophotonics 8(7), 584-596 (2015).

22. C. Bielecki et al., "Classification of inflammatory bowel diseases by means of Raman spectroscopic imaging of epithelium cells," J. Biomed. Opt. 17(7), 076030 (2012).

23. T. Vankeirsbilck et al., "Applications of Raman spectroscopy in pharmaceutical analysis," TRAC Trends Anal. Chem. 21(12), 869-877 (2002).

24. Q. Zhou et al., "Charge transfer between metal nanoparticles interconnected with a functionalized molecule probed by surface-enhanced Raman spectroscopy," Angew. Chem. Int. Ed. Engl. 45(24), 3970-3973 (2006).

25. B. Khlebtsov, V. Khanadeev, and N. Khlebtsov, "Surface-enhanced Raman scattering inside Au@Ag core/shell nanorods," Nano Res. 9(8), 2303-2318 (2016).

26. Y. Fei Chan et al., "Ag dendritic nanostructures as ultrastable substrates for surface-enhanced Raman scattering," Appl. Phys. Lett. 102(18), 183118 (2013).

27. K. Kneipp et al., "Surface-enhanced Raman scattering and biophysics," J. Phys. Condens. Matter 14, R597-R624 (2002).

28. H. Wei and H. Xu, "Hot spots in different metal nanostructures for plasmon-enhanced Raman spectroscopy," Nanoscale 5(22), 10794-10805 (2013).

29. S. Feng et al., "Nasopharyngeal cancer detection based on blood plasma surface-enhanced Raman spectroscopy and multivariate analysis," Biosens. Bioelectron. 25(11), 2414-2419 (2010).

30. S. Feng et al., "Blood plasma surface-enhanced Raman spectroscopy for non-invasive optical detection of cervical cancer," Analyst 138(14), 3967 (2013).

31. D. Lin et al., "Colorectal cancer detection by gold nanoparticle based surface-enhanced Raman spectroscopy of blood serum and statistical analysis," Opt. Express 19(14), 13565-13577 (2011).

32. S. Feng et al., "Study on gastric cancer blood plasma based on surfaceenhanced Raman spectroscopy combined with multivariate analysis," Sci. China Life Sci. 54(9), 828-834 (2011).

33. B. Yan et al., "Label-free blood serum detection by using surfaceenhanced Raman spectroscopy and support vector machine for the preoperative diagnosis of parotid gland tumors," BMC Cancer 15(1), 650 (2015).

34. S. Li, Z. Guo, and Z. Liu, "Surface-enhanced Raman spectroscopy + support vector machine: a new noninvasive method for prostate cancer screening?" Expert Rev. Anticancer Ther. 15(1), 5-7 (2015).

35. O. Petruš et al., "Colloidal lithography with electrochemical nickel deposition as a unique method for improved silver decorated nanocavities in SERS applications," Appl. Surf. Sci. 423, 322-330 (2017).

36. J. Cejkova et al., "Characterization of copper SERS-active substrates prepared by electrochemical deposition," Appl. Surf. Sci. 255(18), 7864-7870 (2009).

37. Y. Li et al., "A template/electrochemical deposition method for fabricating silver nanorod arrays based on porous anodic alumina," Nanomater. Nanotechnol. 7, 1-7 (2017).

38. G. Sandmann, H. Dietz, and W. Plieth, "Preparation of silver nanoparticles on ITO surfaces by a double-pulse method," J. Electroanal. Chem. 491(1-2), 78-86 (2000).

39. M. Ueda et al., "Double-pulse technique as an electrochemical tool for controlling the preparation of metallic nanoparticles," Electrochim. Acta 48(4), 377-386 (2002).

40. T. Zidarič et al., "Multi-pulse galvanostatic preparation of nanostructured bismuth film electrode for trace metal detection," Sens. Actuators B 245, 720-725 (2017).

41. M. Mazur, "Electrochemically prepared silver nanoflakes and nanowires," Electrochem. Commun. 6(4), 400-403 (2004). 
42. L. P. Bicelli et al., "A review of nanostructural aspects of metal electrodeposition," Int. J. Electrochem. Sci. 3(4), 356-408 (2008).

43. R. De Palma et al., "Silane ligand exchange to make hydrophobic superparamagnetic nanoparticles water-dispersible," Chem. Mater. 19(7), 1821-1831 (2007).

44. U. K. Sur and V. Lakshminarayanan, "A study of the hydrophobic properties of alkanethiol self-assembled monolayers prepared in different solvents," J. Electroanal. Chem. 565(2), 343-350 (2004).

45. L. Xue et al., "Advanced SERS sensor based on capillarity-assisted preconcentration through gold nanoparticle-decorated porous nanorods," Small 13(22), 1603947(2017).

46. W. Ren et al., "A simple route for the synthesis of morphologycontrolled and SERS-active Ag dendrites with near-infrared absorption," J. Phys. Chem. C 115(21), 10315-10320 (2011).

47. E. C. Le Ru et al., "Surface enhanced Raman scattering enhancement factors: a comprehensive study," J. Phys. Chem. C 111(37), 13794-13803 (2007).
48. A. G. Lalkhen and A. McCluskey, "Clinical tests: sensitivity and specificity," Contin. Educ. Anaesth. Crit. Care Pain 8(6), 221-223 (2008).

49. D. Lin et al., "Colorectal cancer detection by gold nanoparticle based surface-enhanced Raman spectroscopy of blood serum and statistical analysis," Opt. Express 19(14), 13565-13577 (2011).

50. R. Liu et al., "Surface-enhanced Raman scattering study of human serum on PVA-Ag nanofilm prepared by using electrostatic selfassembly," J. Raman Spectrosc. 42(2), 137-144 (2011).

51. E. Gormally et al., "Circulating free DNA in plasma or serum as biomarker of carcinogenesis: Practical aspects and biological significance," Mutat. Res. 635(2-3), 105-117 (2007).

52. H. W. Han et al., "Analysis of serum from type II diabetes mellitus and diabetic complication using surface-enhanced Raman spectra (SERS)," Appl. Phys. B 94(4), 667-672 (2009).

Biographies for the authors are not available. 